\title{
Transjugular intrahepatic portosystemic shunt via left hepatic - left portal veins
}

\section{Geert Maleux}

University Hospitals Leuven, Belgium

A 38-year-old woman presented with recurrent upper gastrointestinal, variceal bleeding related to non-cirrhotic, portal hypertension associated with veno-occlusive liver disease. Her medical history also included Coats' syndrome and end-stage renal disease.

It was decided to create a transjugular intrahepatic portosystemic shunt (TIPS) to manage the recurrent upper variceal bleedings. However, since access to the right hepatic vein was impossible because of chronic proximal vein occlusion (Fig. 1), the patent's left hepatic vein was cannulated. Puncture from the left hepatic vein into the left portal vein was successful (Fig. 2A) and, after tract balloon dilatation, an expanded tetrafluoroethylene-covered stent (Viatorr, W.L. Gore and Associates, Flagstaff, AZ, USA) was inserted and post-dilated up to $8 \mathrm{~mm}$ diameter (Fig. 2B). Post-interventional follow up showed no variceal bleeding episodes; however, the patient died 1 year later, following bradyarrhythmia and TIPS-induced hepatic encephalopathy.

Conventional TIPS procedures are performed by creating an intraparenchymal tract from the right hepatic vein into the right portal vein. They are associated with a very high success rate [1] related to the relatively large caliber and relatively close anatomical location of the 2 veins. However, in patients with a postoperative anatomy, including right hepatectomy [2] or left liver transplant recipients [3], creation of a TIPS can only be performed via the left hepatic vein. The presented case includes another pathological condition, namely chronic occlusion of the proximal right hepatic vein, which required a left-sided hepatic venous approach to create a portosystemic shunt.

\section{References}

1. Geeroms B, Laleman W, Laenen A, et al. Expanded polytetrafluoroethylene-covered stent-grafts for transjugular

Department of Radiology, University Hospitals Leuven, Belgium

Conflict of Interest: None

Correspondence to: Department of Radiology, University Hospitals Leuven, Belgium, e-mail: geert.maleux@uzleuven.be

Received 20 August 2019; accepted 22 August 2019

Published online 1 October 2019

DOI: https://doi.org/10.20524/aog.2019.0421

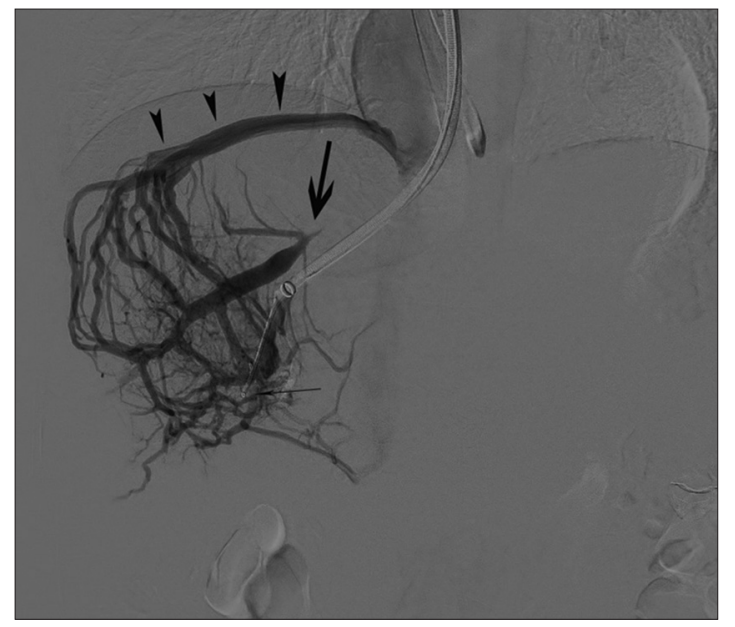

Figure 1 Retrograde injection of iodinated contrast medium into the middle hepatic vein (small arrow) reveals opacification of many veno-venous collaterals and retrograde opacification of an accessory, subdiaphragmatic right hepatic vein (arrows), as well as opacification of the proximally occluded native right hepatic vein (large arrow)
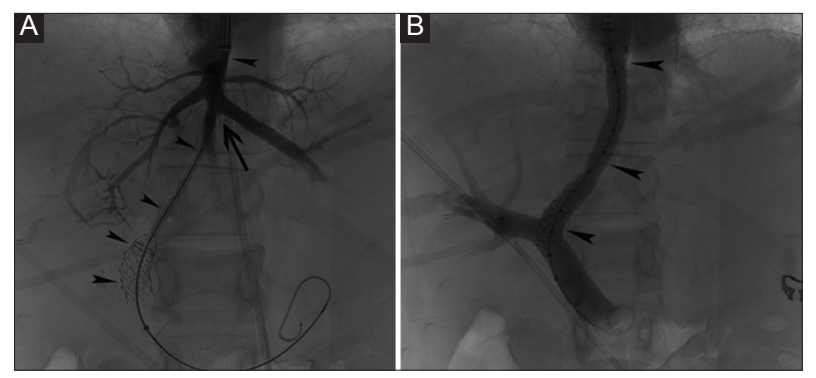

Figure 2 (A) Insertion of the expanded tetrafluoroethylene-covered stent-graft (arrowheads) into the left portal vein through the left hepatic vein (arrow). (B) Completion of direct portography shows preferential flow through the fully patent transjugular intrahepatic portosystemic shunt, placed from the left portal vein up to the confluence of the inferior vena cava and the left hepatic vein

intrahepatic portosystemic shunts in cirrhotic patients: Long-term patency and clinical outcome results. Eur Radiol 2017;27:17951803.

2. Hidajat N, Kreuschner M, Röttgen R, Schröder RJ, Schmidt S, Felix R. Placement of transjugular intrahepatic portosystemic shunt via the left hepatic vein under sonographic guidance in a patient with right hemihepatectomy. Acta Radiol 2003;44:363-365.

3. Saad W, Davies M, Lee D, et al. Transjugular intrahepatic portosystemic shunt in a living donor left lateral liver transplant recipient: technical considerations. J Vasc Interv Radiol 2005; 16:873-877. 